\title{
La pratique musicale, entre l'art et la mécanique. Les effets du métronome sur le champ musical au $\mathrm{XIX}^{\mathrm{e}}$ siècle
}

Musical practice, half way between art and mechanics. The effects of the metronome on the musical field in the $19^{\text {th }}$ century.

Musikalische Praxis zwischen Kunst und Mechanik. Die Auswirkungen des Metronoms auf die Musik im 19. Jahrhundert.

\section{Aurélie Barbuscia}

\section{(2) OpenEdition}

\section{Journals}

Édition électronique

URL : http://journals.openedition.org/rh19/4335

DOI : $10.4000 /$ rh 19.4335

ISSN : 1777-5329

Éditeur

La Société de 1848

Édition imprimée

Date de publication : 31 décembre 2012

Pagination : 53-68

ISSN : 1265-1354

Référence électronique

Aurélie Barbuscia, « La pratique musicale, entre l'art et la mécanique. Les effets du métronome sur le champ musical au XIX ${ }^{2}$ siècle », Revue d'histoire du XIXe siècle [En ligne], 45 | 2012, mis en ligne le 31 décembre 2015, consulté le 01 mai 2019. URL : http://journals.openedition.org/rh19/4335 ; DOI : $10.4000 /$ rh19.4335 


\section{AURÉLIE BARBUSCIA}

\section{La pratique musicale, entre l'art et la mécanique Les effets du métronome sur le champ musical au XIX'e siècle}

En 1815, le métronome apparait au sein de la pratique musicale pour remplir deux fonctions essentielles : servir de référence en matière de tempo à travers l'indication de la vitesse d'exécution d'un morceau et permettre au musicien d'acquérir une précision rythmique par le biais d'un apprentissage progressif. Presque deux siècles après son invention, cet instrument n'a été exploré dans l'historiographie que sous l'angle de l'interprétation musicale et de la légitimité des indications métronomiques 'préconisées' par les compositeurs de la génération beethovénienne ${ }^{1}$ ou encore sous forme d'expérience culturelle du temps confortant l'idée selon laquelle «la musique n'est pas seulement un art dans le temps, mais un art du temps ${ }^{2}$ » Cet article entend revenir sur la rencontre entre la musique et l'objet technique ainsi que sur les tensions que cette rencontre engendre au sein du champ musical. En effet, l'introduction du métronome dans la pratique musicale apparait comme une source incomparable de distinction mettant en évidence une série de scissions - technique/expression, respect/transgression, mécanique/philosophique, pratique/théorie, professionnel/amateur, exécutant/virtuose - qui participent à la professionnalisation du musicien ainsi qu’à une redéfinition des rôles du compositeur, de l'interprète, du virtuose et de l'amateur ainsi que de leurs

1. Un bon nombre d'interprètes et de musicologues se posent encore la question suivante : doit-on ignorer les indications métronomiques "préconisées" par le compositeur sous prétexte qu’elles sont à la limite de l'exécutable? D'où le dernier défi qu'a tenté de relever le chef d'orchestre Riccardo Chailly dans sa récente interprétation des neuf symphonies de Beethoven conforme au tempo échevelé recommandé par le compositeur (Decca, 2011). Aussi, alors que Chailly exécute les symphonies de Beethoven en prenant à la lettre les indications métronomiques - en demi-oscillation - du compositeur, le chef d'orchestre Maximianno Cobra soutient dans sa thèse comme dans son interprétation que le tempo recommandé par Beethoven est deux fois plus lent car ses indications métronomiques, vraisemblablement erronées, correspondraient à une oscillation complète, c'est-à-dire à un aller-retour du balancier. Maximianno Cobra, Les symphonies de Ludwig von Beethoven : une étude analytique, critique et historique en vue d'une nouvelle édition, Thèse de doctorat, Université de Paris Sorbonne, Paris IV, 1999. L'interprétation des autres chefs se situe généralement entre ces deux extrêmes, le tempo tend à être vivace sans pour autant s'en tenir aux valeurs métronomiques indiquées sur la partition. Pour approfondir la question, voir Vincent Arlettaz, "Faut-il jouer Beethoven deux fois moins vite?» in Revue Musicale de Suisse Romande, $\mathrm{n}^{\circ}$ 56-1, mars 2003.

2. Christian Accaoui, Le temps musical, Desclée de Brouwer, Paris, 2001, p. 18. Gisèle Brelet, Le temps musical. Essai d'une esthétique nouvelle de la musique, Paris, PUF, 1949. 
relations respectives. Aussi l'usage du métronome constitue-t-il un enjeu tout au long du XIX ${ }^{\text {e }}$ siècle du fait de l'évolution des conditions socio-économiques du musicien, de la demande nouvelle d'amateurs, de l'avènement de la virtuosité conceptuelle (composition) et technique (exécution) comme de l'éloquence du romantisme et de la mécanisation de la musique, symptomatique de l'ère industrielle.

\section{L'APPARITION DU MÉTRONOME \\ OU L'ILLUSION D'OBJECTIVITÉ EN MUSIQUE}

Dans la musique savante occidentale caractérisée par un complexe système de notation, l'indication du temps a toujours fait l'objet de réflexions, d'expérimentations, d'approches empiriques voire de spéculations donnant naissance à un nombre considérable de signes d'écriture, de méthodes sensorielles et d'objets techniques. Jusqu'au $\mathrm{XI}^{\mathrm{e}}$ siècle, le système de notation musicale ne concerne que la hauteur des sons avant que l'émergence de la polyphonie ne rende nécessaire la mise en place d'une représentation graphique du rythme à durées proportionnelles. Faute de régler le problème de la mesure du temps, l'avènement au XVe siècle d'un rythme dit "mesuré ${ }^{3}$ " finit par en poser de nouveaux. C'est à partir de là que se creuse le clivage entre une écriture musicale de plus en plus sophistiquée et une exécution incapable de répondre à toutes les exigences annotées. En dépit de son perfectionnement, l'écriture musicale ne permet pas de faire, de la lecture de la partition, un déchiffrage au moins fidèle si ce n'est intact de l'œuvre pensée et créée par le compositeur ${ }^{4}$. Le musicien est alors amené à chercher des solutions en dehors de la partition afin de combler les lacunes de la transmission écrite. Les différentes solutions proposées - qu'il s'agisse de l'élaboration de méthodes sensorielles comme de l'invention d'objets techniques - sont intimement liées au contexte épistémologique dans lequel ces dernières voient le jour. Aussi, en parallèle des grandes innovations dans le domaine médical, nombreuses sont les méthodes qui consistent à faire de la mesure du temps musical une perception physique ayant pour fondement les activités humaines les plus fréquentes comme la marche ou la danse, voire les fonctions vitales telles que le rythme cardiaque ou la respiration. Le manuel d'orchésographie ${ }^{5}$, recueillant toutes les danses pratiquées au $\mathrm{XVI}^{\mathrm{e}}$ siècle, consiste ainsi à régler le mouvement musical sur celui du pas de marche et de danse. Les danses telles que l'allemande, la courante, la

3. Le temps musical se voit dés lors soumis à la périodicité à travers l'introduction d'une norme conceptuelle (le mètre) dont la barre de mesure représente l'archétype.

4. Françoise Escal, Espaces sociaux, espaces musicaux, Lausanne, Payot, 1979.

5. Thoinot Arbeau, anagramme de Jehan Tabourot, L'Orchesographie et traicté en forme de dialogue par lequel toutes personnes peuvent facilement apprendre \& practiquer l'honneste exercice des dances, privilège daté du 22 novembre 1588, publié en 1589 . 
gigue et la sarabande - pour ne citer qu'elles - finissent par se fixer dans les mémoires au point de faire office d'indication rythmique et de renvoyer directement à un mouvement ainsi qu'à une mesure spécifiques. Seulement, les pièces instrumentales étant pour la plupart dépourvues d'intention chorégraphique, l'usage des danses comme empreinte rythmique se perd peu à peu pour des raisons esthétiques autant que d'innovation musicale ${ }^{6}$. Un autre procédé selon lequel «on peut par le moyen des battemens du pouls, réduire chaque sorte de mesure à son juste mouvement ${ }^{7}$ » est mis au point par un flûtiste allemand en 1752 mais cette méthode physiologique se voit bientôt abandonnée au profit de la nouvelle technique capable de gagner en précision et de dépasser l' «à-peu-près ${ }^{8}$ ".

Dans le cadre de l'établissement d'un nouveau paradigme scientifique, le musicien fait appel au mécanicien afin qu'il invente «une machine qui put fournir au compositeur le moyen de fixer pour l'avenir, avec une rigueur mathématique, l'indication du mouvement, tel qu'il le sentait et le voulait ${ }^{9} »$. La mesure du temps musical bénéficie ainsi des progrès accomplis dans le domaine de l'horlogerie, eux-mêmes révélateurs d'une évolution dans la perception et l'intériorisation du temps au quotidien. Seulement, si les divergences entre mesure et rythme, entre écriture et exécution, entre notation et pratique s'accroissent au XVe siècle avec l'instauration d'une horloge interne à laquelle se superposent les fluctuations du rythme (le rythme mesuré), le recours à l'objet technique, entendu comme horloge externe et comme éloge à l'objectivité calculatoire, ne peut qu'accentuer le problème. Le premier objet technique capable de fixer la valeur des durées est mis à la disposition du musicien-praticien à l'aube du XVIII siècle. Il s'agit du chronomètre conçu par l'instrumentiste Etienne Loulié (1698) et promu comme le seul instrument capable d'indiquer avec exactitude le tempo des airs de Lully, jouissant alors d'une forte popularité ${ }^{10}$. Plusieurs physiciens européens cherchent tout au long du XVIII ${ }^{e}$ siècle à perfectionner le chronomètre de Loulié $^{11}$ avant d'aboutir à l'invention du métronome, pendule enfermé dans une boite pyramidale. L'allemand Johann Maëlzel, mécanicien de la cour de

\footnotetext{
6. Seul le minuetto dans les sonates et les symphonies perdure.

7. Johann Joachim Quantz, Essai d'une méthode pour apprendre à jouer de la flute traversière : avec plusieurs remarques pour servir au bon gout dans la musique le tout éclairci par des exemples et par XXIV. Tailles douces, Chez Chrétien Frederic Voss, 1752, p. 267 et Versuch einer Anweisung die Flöte traversiere zu spielen, Berlin, 1752.

8. François-Joseph Fétis, La musique mise à la portée de tout le monde, Paris, Mesnier, 1830, p. 40.

9. Louis-Casimir Colomb, La musique, Paris, Hachette, 1878, p. 51.

10. Étienne Loulié, Eléments ou Principes de musique, Pars, Ballard, 1996, p. 88, cité par Jean Duron, Le prince et la musique : les passions musicales de Louis XIV, Wavre, Éditions Mardaga, 2009, p. 299.

11. Laffilard, Sauveur, Gabory, Pelletier, Renaudin, Bréguet et Galin en France, les chantres Weisske de Meissen, Stackel de Burg, Crotch et Harrison en Angleterre, Burja de Berlin, Gottfried Weber en Allemagne et Winkel à Amsterdam. Louis-Félix Despréaux, nommé Jean-Étienne, publie en 1817, un tableau des mouvemens de la musique sous le nom de Chronomètre Musical établi sur les bases du pendule astronomique, cité par François-Joseph Fétis, Biographie universelle des musiciens et bibliographie générale de la musique, Paris, Leroux, 1836, vol. 3, p. 296 et François-Joseph Fétis, La musique..., op. cit., p. 42.
} 
l'empereur d'Autriche, aussi connu pour ses inventions spectaculaires (l'orchestre mécanique, la poupée parlante ou le trompette automate) que pour ses supercheries (le joueur d'échec mécanique ${ }^{12}$ ), s'approprie la paternité de la découverte de Dietrich Nikolaus Winkel et obtient à Paris le brevet d'invention du métronome (en 1815) ${ }^{13}$. Force est de constater que nonobstant l'usurpation de la priorité d'invention, les noms de Maëlzel et du métronome sont rapidement rendus indissociables par l'inscription des lettres "M. M.» (Métronome de Maëlzel) placées en tête des morceaux de musique. En effet, si le métronome apparait, en tant qu'objet technique, au sein de la pratique musicale et dans le quotidien de l'interprète, c'est parce qu'il a été au préalable reconnu par le compositeur et intégré - sous forme d'indication métronomique - au sein de l'écriture musicale. Autrement dit, à mesure que son usage se répand, l'objet technique conçu pour compenser les insuffisances du système de notation musicale se voit en contrepartie institué et légitimé par ce même système. Paradoxalement, malgré les appareils tels que le chronomètre ou le rythmomètre ${ }^{14}$ ayant précédé sa construction, le métronome est, dès son apparition, présenté comme une découverte sans précédent. La description suivante représente l'interprétation canonique d'une recherche ayant abouti à la découverte historique du métronome, instrument voué à "faire époque dans l'empire de la musique ${ }^{15}$ » comme l'écrit le compositeur Henri Montan Berton et sur lequel reposent tous les espoirs :

On a recherché l'origine du sentiment rythmique dans la marche, dans les battements du cœur, dans les bruits de la respiration, et plus mathématiquement dans les oscillations invariablement isochrones du pendule. [...] Le métronome, instrument qui mesure le rythme musical comme le monocorde mesure les vibrations, n'est pas autre chose qu'une horloge qui bat les fractions de minute, comme le ferait un pendule dont on pourrait à volonté faire varier la longueur ${ }^{16}$.

Comme l'exprime le brevet d'invention, c'est la singularité du métronome qui justifie son approbation : «Le dessin d'application que M. Maëlzel a joint à la demande d'un brevet d'invention de 5 ans pour un métronome, nom qu'il donne à une espèce de chronomètre, suffisent pour faire distinguer cet instrument de tout ceux imaginés et construits jusqu’à ce jour pour le même

12. INPI, 1BA1907.

13. Archives nat., F12 1024 A. INPI, 1 BA883, délivré le 14 septembre 1815. Pour une description du métronome, voir Adolphe Benestor Lunel [dir.], Dictionnaire universel des connaissances humaines..., Paris, Lacroix-Comon, Tome 6, 1857-59, p. 102. Dietrich Nikolaus Winkel, en 1818 réclame la priorité d'invention dans un article de la Gazette musicale de Leipzig, l'Institut des Pays- Bas tranche le différend en sa faveur.

14. Le rythmomètre est inventé en 1782 par Philippe-Auguste Duclos (horloger de Paris) puis approuvé par les professeurs de l'école royale de chant de Paris à la tête desquels se trouve le compositeur FrançoisJoseph Gossec.

15. BnF, Musique, La. 69 (Début), Bob 21341, n. 195.

16. Albert Lavignac, La musique et les musiciens, Paris, Delagrave, 1938, p. 74. 
objet $^{17}$.» En réalité, la principale originalité du métronome réside dans le fait que le frappé de chacune des vibrations du balancier est perceptible à l'oreille. Son tic-tac implacable fait l'objet d'une ultime amélioration en 1829 qui consiste à accentuer le premier temps de chaque mesure au moyen d'une augmentation sensible de son niveau d'intensité sonore ${ }^{18}$.

\section{LA PROMOTION DU MÉTRONOME}

À LA MESURE DU MARCHÉ MUSICAL

Dès l'obtention du brevet grâce auquel les compositeurs Cherubini, Méhul, Spontini, Paër, Boieldieu, Berton, Gossec, Persuis, Catel, Martini, Pleyel et Lesueur ont pris connaissance de l'existence du métronome, Maëlzel s'empresse de fonder en 1816 une société de fabrication à Paris afin de diffuser son nouvel instrument dans les plus brefs délais. En 1817, ce dernier retourne, non sans raison, à Vienne où il a su, en qualité de mécanicien de la cour, créer, entretenir et développer un ample réseau de connaissances cantonné au monde musical. En effet, durant la première partie de sa carrière, Maëlzel faisait partie de l'entourage du prince Lichnowsky, l'un des principaux mécènes de Beethoven. Le grand maître s'était d'ailleurs lié d'amitié avec le mécanicien au point de lui dédier une œuvre symphonique - Wellingtons Sieg, op. $91^{19}(1813)$ - destinée à la promotion de son orchestre mécanique connu sous le nom de panharmonicon ${ }^{20}$. En guise de remerciement, le mécanicien n'avait pas hésité à déposséder Beethoven de sa propriété intellectuelle en utilisant à loisir son morceau pour promouvoir sa machine à Londres ${ }^{21}$. On peut donc imaginer que la réputation d'usurpateur que Maëlzel s'est taillée à Vienne comme à Londres le précède lorsque la paternité controversée du métronome alimente de nouvelles polémiques. Seulement, pour garantir au métronome un certain succès, le mécanicien se sait tributaire du bon vouloir des compositeurs. Pour ce faire, il est indispensable de regagner la confiance de Beethoven faisant figure d'autorité et de référence dans le monde musical de l'époque. Le mécanicien commence donc par réclamer le

17. INPI, 1 BA883, Brevet d'invention de 5 ans, instrument ou mécanique nommé métronome, propre à marquer les mesures dans la musique, 1815, Maelzel Johann Nepomuk, Séance du 26 août 1815.

18. INPI, 1 BA3178, Brevet d'invention et de perfectionnement de 5 ans, divers changemens faits au mécanisme primitif du métronome, 1829. 218-014, 218-015. Collection complète des lois, décrets d'interee général, traités internationaux, arretés, circulaires, instructions, etc., France, Recueil Sirey, 1833, vol. 32, Louis-Philippe $1^{\text {er }}, 10$ juillet 1832, p. 384.

19. Le second mouvement de la $8^{\mathrm{e}}$ symphonie de Beethoven s'en inspire.

20. Maëlzel avait déjà obtenu à Paris en 1807 une pièce du compositeur Luigi Cherubini intitulée écho pour le même instrument, voir Paul Metzner, Crescendo of the Virtuoso: Spectacle, Skill and Self-promotion in Paris during the Age of Revolution, Berkeley / Los Angeles / London, University of California Press, 1998, p. 182-188.

21. La déposition de Beethoven ainsi que le verdict du tribunal sont soigneusement retranscrits dans : Anton Felix Schindler et Donald MacArdle, Beethoven as I knew him, Mineola NY, Courier Dover Publications, 1966, p. 486-489. 
soutien des compositeurs en envoyant un exemplaire du métronome à deux cent d'entre les plus réputés. Curieusement, Beethoven est l'un des premiers à répondre à son appel en publiant une table comprenant les indications métronomiques qu'il recommande - dès lors - pour l'exécution de ses huit symphonies $^{22}$. Le métronome lui semble un moyen efficace pour suppléer aux faiblesses du système de notation musicale en vigueur, notamment «la terminologie italienne» :

Relativement aux indications de tempo, [...] qu'y a-t-il de plus absurde que [le terme] Allegro, qui signifie une fois pour toutes joyeux, alors que nous sommes si éloignés du sens de cette indication, de telle sorte que le morceau lui-même dit le contraire de l'indication. [...] quant à moi, $\mathrm{j}$ 'ai imaginé depuis longtemps renoncer à ces appellations absurdes Allegro, Andante, Adagio, Presto; le métronome de Maelzel nous en donne la meilleure occasion ${ }^{23}$.

En bon stratège, Maëlzel tente également de solliciter M. Pleyel qui, en tant que compositeur, fondateur de la plus productive marque de piano de France ${ }^{24}$, membre de l'Institut de France et éditeur, a toutes les raisons d'être digne d'intérêt :

J'ai l'honneur de vous adresser ci-joint deux billets d'entrée à mon salon d'exhibition d'objet mécanique dans l'espoir [...] qu'après avoir assisté à l'une de mes séances vous aurez la complaisance d'en dire quelques mots dans votre journal [...] que vous fassiez annoncer ce spectacle en même temps que les autres ${ }^{25}$.

Promu par les compositeurs comme le seul « remède $^{26}$ » efficace à un mal auquel l'art musical n'a jamais su obvier, le métronome passe, sans difficulté ni phase intermédiaire, de l'accessoire faisant le bonheur de quelques-uns à l'objet indispensable à toute pratique musicale.

Le succès du métronome de Maelzel et sa grande utilité sont reconnus depuis longtemps des suffrages des savans, et des premiers artistes de toutes les capitales de l'Europe ont assuré pour toujours l'usage de cet instrument à la fois simple et peu coûteux. Les compositeurs de tous les pays ayant contracté l'habitude de cotes en

22. Allgemeine Musikalische Zeitung de Leipzig, le 17 décembre 1817.

23. Sieghard Brandenburg (éd.), Ludwig von Beethoven. Briefurechsel, Gesamtausgabe, München, Henle, 1996, p. 130-131. Lettre $\mathrm{n}^{\circ} 1196$ selon l'édition Henle, novembre 1817, à Ignaz Franz von Mosel, $\mathrm{n}^{\circ} 845$ selon l'édition de Emily Anderson (1961).

24. Parmi les marques présentes sur le marché français avant 1850, Pleyel est la plus productive en France à raison de 800 pianos par an en 1850, cf. Cyril Ehrlich, The Piano: a History, Oxford, Clarendon Press, 1990, p. 110.

25. BnF, Musique, La. 69 Bob 21341, n. 309, Lettre de Maelzel à Pleyel, Paris, le 8 mai 1821.

26. BnF, Musique, La. 69 (Début) Bob 21341, n. 195, rapport rédigé par Henri Montan Berton. 
marge de leur musique [...] il en résulte que ce mécanisme devient indispensable à tous les musiciens... ${ }^{27}$

Le rapport de l'Institut de France - par définition laudatif - va même jusqu'à avancer que "le métronome est à la musique ce que la boussole est à la navigation ${ }^{28}$ ", tandis qu'un nombre considérable de discours promotionnels encourageant son usage tendent à l'associer à l'indétrônable diapason ${ }^{29}$. Le premier, utile au travail rythmique, et le second, appliqué au travail d'intonation, sont invités à faire tour à tour partie intégrante de l'équipement mais aussi du quotidien de tout musicien. En d'autres termes, l'ample diffusion de l'un finit par aller de pair avec la promotion de l'autre. Les méthodes de solfege et de théorie musicale contribuent d'ailleurs à la construction d'un lien privilégié entre les deux accessoires puisqu'elles en revendiquent la complémentarité et recommandent aux musiciens d'en faire un usage rapproché $^{30}$. Ainsi, dès l'année 1817 , le métronome circule dans toute l'Europe et s'impose rapidement sur le marché en se présentant comme le concurrent d'autres instruments de mesure du temps musical. En dépit de la préférence accordée à la nouvelle invention du métronome, son ancêtre, le chronomètre, continue d'occuper une place importante sur le marché car on lui reconnaît "le double avantage d'être une construction simple et peu dispendieuse ${ }^{31}$ ». Outre les critères techniques, le métronome et le chronomètre se distinguent vraisemblablement par leur prix, déterminant par là même l'origine sociale de leur utilisateur.

Parmi les instruments inventés pour mesurer le temps en musique, j’indiquerai seulement : 1. Le chronomètre de Galin, [...] Il est d'une simplicité extrême et ne coûte que quelques centimes.; 2. Le métronome de Maelzel, petit instrument fort commode [mais] d'un prix assez élevé. Ce dernier sera l'instrument du riche; celui de Galin sera celui du pauvre ${ }^{32}$.

Dans le rapport rédigé en 1815 par Henri Montan Berton, membre de la section musique de l'Académie française ${ }^{33}$, le prix du métronome est censé être accessible à toutes les bourses. Pour donner un ordre d'idée tout en restant dans le cadre de la vie musicale parisienne, un métronome ordinaire revient à 25 francs tandis que le prix d'un billet dans l'un des théâtres parisiens les plus

27. INPI, 1 BA3178, Brevet d'invention et de perfectionnement de 5 ans, divers changemens faits au mécanisme primitif du métronome, 1829, 216-007.

28. BnF, Musique, La. 69 (Début), Bob 21341, n. 195.

29. Inventé par l'Anglais Shore en 1711.

30. «Conseils aux commençants pour étudier seuls un air. Ici, comme nous l'avons toujours fait, il faut séparer l'étude de l'intonation de celle de la mesure, pour réunir ensuite ces deux choses. " Émile et Nanine Chevé, Méthode élémentaire de musique vocale, Paris, Chevé, 1857, p. 104.

31. François-Joseph Fétis, La musique ..., op. cit., chapitre 6.

32. Émile et Nanine Chevé, op. cit., p. 254.

33. BnF, Musique, La. 69 (Début) Bob 21341, n. 195, rapport rédigé par H. M. Berton. 
réputés - l'Académie royale de musique ou le Théâtre-italien - s'échelonne de 2 à 10 francs en fonction de la place occupée ${ }^{34}$.

Guide de prix des instruments de musique et autres prestations musicales en $1835^{35}$

\begin{tabular}{|l|c|}
\hline \multicolumn{1}{|c|}{ Instruments ou prestations musicales } & Prix en francs \\
\hline Métronome ordinaire & 25 \\
\hline Métronome marquant les premiers temps de chaque mesure & 40 \\
\hline Piano carré ou piano droit & de 650 à 900 \\
\hline Violon (bon patron de Mirecourt) & 50 \\
\hline Guitare (commune de Mirecourt) & de 20 à 40 \\
\hline Clarinette & 125 \\
\hline Hautbois & 120 \\
\hline Cor complet avec coulisse & 150 \\
\hline Leçon de musique ou d'instrument (par leçon) & de 3 à 20 \\
\hline Location d'un piano (par mois) & de 5 à 30 \\
\hline Location d'un violon (par mois) & de 3 à 10 \\
\hline
\end{tabular}

Ce tableau nous permet de remettre en cause l'idée selon laquelle «la modestie de son prix [métronome] met à même de se le procurer toutes les personnes qui s'occupent de la musique ${ }^{36}$ » en faveur du présent constat : le métronome "sera l'instrument du riche" tandis que le chronomètre «sera celui du pauvre ${ }^{37} »$. Pour convaincre de la nécessité de perfectionner le mécanisme du métronome (1829), l'horloger Jean Wagner n’hésite pas à faire miroiter une sensible diminution de son prix, susceptible d'en démocratiser l'usage : «le perfectionnement de l'invention de Maelzel permettra de l'accorder au public à moitié prix ce qui le met à la portée des artistes ${ }^{38}$." Aussi longtemps que persiste le décalage entre les discours de promotion et la réalité pratique de l'objet, entre sa valeur marchande et sa valeur symbolique, l'usage du métronome reste socialement déterminé.

La promotion du métronome ne représente pas uniquement un enjeu économique et social mais s'inscrit également dans la "politique culturelle» de la seconde Restauration. Il s'agit donc d'interroger les rôles joués par l'Académie royale des Beaux-Arts ainsi que par le pouvoir politique dans le processus de mise à jour, de sélection et de mise en circulation des objets techniques

34. Almanach des spectacles, 1830, p. 33-39.

35. Nous avons tenté de réunir les instruments de musique les plus joués ainsi que les prestations musicales les plus courantes de l'époque. Ce tableau permet de situer la valeur commerciale du métronome par rapport à celle d'autres instruments. Nous tenons à indiquer le montant du piano à la vente comme à la location car il existe un lien privilégié entre l'usage du métronome et la pratique du piano : cf. Planque, Agenda musical : contenant les noms et demeures de tous les artistes et commerçans en musique, Paris, Collinet, 1835, p. 270-285.

36. BnF, Musique, La. 69 (Début) Bob 21341, n. 195, rapport rédigé par H. M. Berton.

37. Émile et Nanine Chevé, op. cit., p. 254.

38. INPI, 1 BA3178, Brevet d'invention et de perfectionnement de 5 ans, divers changemens faits au mécanisme primitif du métronome, 1829, 218-017. Lettre écrite par Jean Wagner, mécanicien du roi, le 30 mai 1832. 
au service du musicien. En effet, outre le fait d'adopter une approche scientifique et théorique de la musique par le biais de dissertations et de réflexions sur l'art musical, la section de Composition musicale de l'Institut de France joue un rôle actif dans le processus arbitraire de classement, déclassement et reclassement ${ }^{39}$ des objets techniques inhérents à la pratique musicale ou encore dans l'amélioration de la facture d'instruments musicaux s'accompagnant souvent de la publication de méthodes musicales. Il faut, tout d'abord, rappeler que l'Institut de France entretient une étroite relation avec le pouvoir politique dans la mesure où ce dernier lui assure protection et pérennité. En ce sens, la mise sur le marché de nouveaux objets techniques nécessite l'approbation des détenteurs du pouvoir intellectuel, eux-mêmes tributaires du bon vouloir du pouvoir politique. Le pouvoir de l'un légitimant celui de l'autre, les membres de l'Institut font systématiquement allusion au ministre de l'Intérieur pour se justifier, en amont, de la mise à l'examen comme de l'approbation d'un objet technique auquel le pouvoir politique délivre, en aval, un brevet d'invention ${ }^{40}$. Les raisons pour lesquelles certaines améliorations en matière de facture musicale sont adoptées méritent notre attention. Nous nous contenterons ici de citer l'une des plus caractéristiques : "En raison des services qu'elle peut rendre aux corps de musique militaire de notre cavalerie pour qui les trompettes sont ce que les tambours sont à l'infanterie, nous proposons à l'Académie d'accorder à l'auteur l'honorable appui de son approbation ${ }^{41}$ ». Ce rapport montre que la promotion de la nouvelle technique renvoie souvent à une pratique utilitariste de la musique allant à l'encontre de la virtuosité de l'artiste. De fait, le poids accordé aux notions d'utilité publique, de facilité et de philanthropie au sein des procès-verbaux de l'Académie des Beaux-Arts ${ }^{42}$ laisse à penser que les nouveaux objets techniques s'adressent principalement aux pratiques amateurs ${ }^{43}$.

La double fonction théorique (érudition/réflexion) et pratique (sélection d'instruments techniques/évolution de la facture instrumentale) dévolue à la section de Composition musicale de l'Académie s'exerce parfois de manière complexe voire contradictoire. Le métronome, objet technique mis au service de la pratique musicale, donne ainsi lieu à un débat théorique. Le compositeur et membre de l'Institut Henri Montan Berton, chargé de rédiger le

39. Pierre Bourdieu, "Classement, déclassement, reclassement", Actes de la recherche en sciences sociales, vol. 24 , novembre 1978 , p. $2-22$.

40. Béatrice Bouvier et François Fossier, Procès-verbaux de l'Académie des Beaux-Arts, Jean-Michel Leniaud [dir.], Tome troisième, 1821-1825, Paris, École des Chartes, 2003.

41. Archives A.B.A., 5 E 1822, dossier «Procès-verbaux-rapports", 1822, rapport à l'Académie des beauxarts de l'Institut royal de France sur une méthode de trompette à clefs daté du 7 décembre; dossier "Lettres des ministres ", 9 octobre, signée du chef de la $4^{\circ}$ division.

42. Béatrice Bouvier et François Fossier, Procès-verbaux..., op. cit.

43. Outre les pratiques amateurs dont il est ici question, les améliorations effectuées dans la facture instrumentale participent à l'évolution même du concept de virtuosité en musique. L'article suivant montre combien la notion de virtuosité est tributaire de l'évolution de l'organologie au cours des siècles : cf. Geneviève Bernard-Krauss, "La virtuosité dans l'œuvre de Tartini et Galeazzi» in Anne Penesco [dir.], Défense et illustration de la virtuosité, Lyon, Presse universitaire de Lyon, 1997. 
rapport visant à louer les mérites de cet instrument, sera également l'auteur, quelques années plus tard, d'une dissertation intitulée De la musique mécanique et de la musique philosophique ${ }^{44}$ (1821) adressée aux membres de l'Institut. Dans cette dissertation, Berton fait état de son mépris à l'égard d'une musique dite "mécanique" représentée par le compositeur italien à succès : Rossini. Étrangement, Berton tente d'affermir ses conceptions esthétiques en relatant l'une de ses conversations avec Maëlzel au cours de laquelle le mécanicien prend à son tour position contre la musique rossinienne. Si Maëlzel devait créer une machine à composer, elle ne serait, selon lui, capable que de composer du Rossini puisqu'il s'agit d'un "art industriel» qui sacrifie l'expression au profit de "l'effet ${ }^{45}$ » et marque, par là même, la décadence de l'art musical. Le caractère conservateur de l'esthétique musicale homologuée par les membres de l'Académie des Beaux-Arts nous permet de remarquer le net décalage entre l'actualité de l'Académie et la réalité de la création artistique à travers la promotion - a posteriori - d'un style compositionnel. Si l'innovation technique pose problème en termes de composition musicale, la nouvelle technique jouit en revanche d'un meilleur accueil sous la Coupole de l'Institut de France lorsqu'il s'agit du perfectionnement de la facture instrumentale ou de la mécanisation de la musique. Finissons par observer qu'au-delà de sa stricte utilité pratique, l'objet technique peut faire office de matière voire de matériau : matière à réflexion en soulevant un débat théorique tel que l'illustre la dissertation de Berton ou matériau de base de la création artistique, pensons notamment au canon de Beethoven paraphrasant le tic-tac du métronome "Ta ta ta, lieber Mälzel ${ }^{46}$ ».

\section{Pour Une INTERPRÉTATION MUSICALE « MESURÉE »}

Comme son nom l'indique, la section de Composition musicale de l'Institut de France vise à réunir un cénacle d'artistes et à saluer leur double talent de compositeurs de haut vol et de savants théoriciens (professeur de composition musicale et/ou d'harmonie). Autrement dit, ce n'est pas au praticien qu'il incombe de régir l'évolution de la pratique musicale à travers l'approbation d'objets techniques ou de transformations dans la facture musicale même si, dans bien des cas, l'Académie se voit forcée de faire appel aux compétences spécifiques de l'interprète pour juger de l'intérêt de tel ou

44. BnF, Musique, $8^{\circ} \mathrm{B}$ pièce 218, Henri Montan Berton, De la musique mécanique et de la musique philosophique in l'Abeille, de l'Institut, Paris, 1826. «Séance du samedi 14 juillet 1821 » in Béatrice Bouvier et François Fossier, Procès-verbaux de l'Académie des beaux-arts, Jean-Michel Leniaud [dir.], Tome 3, 1821-1825, Paris, École des Chartes, 2003, p. 40.

45.Henri Montan Berton, De la musique mécanique et de la musique philosophique, Paris, Alexis Eymery libraire, 1826, p. 36.

46. Ce canon (WoO 162) pourrait également s'apparenter au second mouvement de la Symphonie $n^{\circ} 101$ en ré majeur "l'horloge» de Joseph Haydn. Parmi les œuvres plus récentes, citons l'emblématique poème symphonique pour 100 métronomes de Ligeti (1962). 
tel perfectionnement ${ }^{47}$. Pour ce qui est de l'introduction du métronome, les décisions sont prises sans que l'avis de l'interprète ne soit recueilli et l'instrument est approuvé sans que le rapport ne fasse en aucune manière allusion aux bénéfices que l'interprète peut en tirer. Nous pouvons ainsi avancer que les compositeurs s'expriment ici en faveur du métronome pour des raisons inhérentes à leur propre catégorie professionnelle.

La déclaration authentique faite par les compositeurs les plus célèbres de l'Europe, de ne plus indiquer la mesure de leurs ouvrages que par les divisions métronomiques nous est un sûr garant que la musique que nous entendons à Paris, bien que composée à Vienne, à Milan ou à Constantinople sera bien celle de l'auteur avec les intentions qu'il a eu [...]. C'est ainsi que les chefs d'œuvre parviendront davantage et que la postérité sera redevable à M. Maëlzel. J'entends après des siècles les beaux morceaux de nos grands maitres comme si eux même conduisaient l'orchestre ${ }^{48}$.

Les raisons invoquées ci-dessus laissent entendre que le sujet-créateur ambitionne de renforcer son contrôle sur la matière même de son travail en exerçant davantage d'autorité sur l'interprète, invité à restituer le plus fidèlement possible ses intentions originelles ${ }^{49}$. Comme un traité sur la musique remontant à la fin du XIX siècle l'atteste : en l'absence de métronome, «le champ [musical] rest[e] encore trop ouvert aux interprétations individuelles des exécutants ${ }^{50}$ ", ce qui implique que l'interprète dispose d'une excessive marge de manœuvre susceptible de mettre en péril la postérité de l'ouvrage. Il s'agit ici de destituer l'interprète de son rôle de co-auteur, de se prémunir de son éventuelle action sur l'ouvrage afin d'éviter que «l'intention du compositeur" ne soit " dénaturée dans l'exécution ${ }^{51}$ ". Les compositeurs plaident donc en faveur d'une identification savante de l'œuvre musicale où la trace écrite et son interprétation sont censées confluer. Dans ces conditions, l'œuvre musicale, quoique par essence inachevée, est assimilée à un objet clos. La mise en place de ce nouvel ordre hiérarchique que les discours promotionnels ne manquent pas de mettre en évidence devient la pierre angulaire sur laquelle repose le succès du métronome auprès des compositeurs : «l'avantage qui résulte de cette machine est telle que l'Auteur en musique peut dire à son correspondant muni du métronome de faire exécuter un morceau de musique

47. Ce rapport illustre fort bien le problème : Archives A.B.A., 5 E 12, 1821, dossier «Procès-verbauxrapports", rapport fait à l'Académie des Beaux-arts de l'Institut royal de France au nom de sa section de musique sur les procédés employés sur M. Andrea Antonin Schmittschneider, pour le perfectionnement de l'instrument de musique appelé «cor ", voir «Séance du 28 juillet 1821 » in Béatrice Bouvier et François Fossier, Procès-verbaux..., op. cit., p. 45.

48. BnF, Musique, La. 69 (Début) Bob 21341, n. 195.

49. Pierre-Michel Menger, "Le travail à l'œuvre, Enquête sur l'autorité contingente du créateur dans l'art lyrique», Annales, Histoire, Sciences Sociales, 2010-3.

50. Louis-Casimir Colomb, op. cit., p. 50.

51. François-Joseph Fétis, La musique..., op. cit., p. 38. 
à cinquante ou cent degrés de vitesse pour la mesure ${ }^{52} »$. Le remplacement du mot "interprète» par celui d' "exécutant», lorsqu'il est question du métronome, témoigne de l'impact qu'exerce l'appareil sur le statut même de l'interprète. Le terme d'exécutant est, en effet, le signe d'une dévalorisation du rôle de l'interprète au profit de celui du compositeur. L'exécution tend ainsi à substituer le jeu et à limiter l'interprétation. La rature que présente ce rapport en est l'exemple le plus frappant : «Avec le métronome on ne marche plus au hasard dans l'exécution; les chef-d'œuvre [sic] de nos grands maitres seront joués exécutés avec l'inspiration de leur génie et les compositeurs auront un point fixe pour indiquer le mouvement de leur musique ${ }^{53}$ ». L'image paternelle de l'auteur-créateur ne fait qu'encourager la "subalternité de l'artiste-musi$\operatorname{cien}^{54} »$.

Au cours de la seconde moitié du XIX ${ }^{e}$ siècle, l'émergence d'un nouvel acteur au sein de la vie musicale, le chef d'orchestre-interprète et non compositeur, ne fait que consolider cette relation filiale compositeur/interprète tout en faisant du respect des instructions métronomiques l'un des principaux postulats sur lequel se fonde la relation. C'est ce dont Hector Berlioz fait état en 1855 dans son traité sur l'art de diriger l'orchestre :

Le chef, avant tout, est tenu de posséder une idée nette [...] de l'œuvre dont il va diriger l'exécution $[\ldots]$ pour pouvoir, sans hésitation ni erreur, déterminer dès l'abord les mouvements voulus par le compositeur. S'il n'a pas été à même de recevoir directement de celui-ci ses instructions [...] il doit recourir aux indications $\mathrm{du}$ métronome [...], la plupart des maittres ayant aujourd'hui le soin de les écrire en tête et dans le courant de leurs morceaux ${ }^{55}$.

Nombreux sont ainsi les chefs d'orchestre soucieux de montrer aux compositeurs avec quel zèle ils ont suivi leurs consignes comme en témoigne la lettre suivante que Fétis ${ }^{56}$ adresse à Hiller : "Je vous ai regretté hier [...] vous auriez été, je crois, pleinement satisfait de l'exécution de votre symphonie [...]. Les mouvements que vous m'avez indiqués par le métronome sont ceux que j'avais pris " 57 . L'inverse est tout aussi vrai, en ce sens que les compositeurs ne manquent pas de recommander leurs indications métronomiques et d'exercer leur mainmise sur l'interprétation des chefs : «vous aurez la bonté

52. INPI, 1 BA883, Brevet d'invention de 5 ans, instrument ou mécanique nommé métronome, propre à marquer les mesures dans la musique, 1815, Maelzel Johann Nepomuk, séance du 26 août 1815.

53. BnF, Musique, La. 69 (Début) Bob 21341, n. 195.

54. Joseph-Marc Bailbé, "De Liszt à Berlioz : réflexions sur la condition de l'artiste-musicien ", Romantisme, $1987, \mathrm{n}^{\circ} 57$, p. 8.

55. Hector Berlioz, "Le chef d'orchestre : théorie de son art" in Grand Traité d'instrumentation... op. cit., 1855.

56. François-Joseph Fétis, «Du respect du Directeur de musique ou du Chef d'Orchestre pour les œuvres des compositeurs" in Manuel des compositeurs, directeurs de musique, chef d'orchestre et de musique militaire, Paris, Brandus, 1837, chapitre 7, p. 127-130.

57. «Lettre de Fétis à Ferdinand Hiller [57-12], Bruxelles, 11 mai 1857 » in François-Joseph Fétis, Correspondances, Wavre, Éditions Mardaga, 2006, p. 393. 
de vous intéresser, écrit Lvoff à Fétis, à l'exécution, et surtout aux mouvements que j’ai indiqué par le métronome de Maeltzel ${ }^{58}$.

\section{LE MÉTRONOME : UN OBJET PARTAGÉ À L'USAGE DIVERSIFIÉ}

À l'heure où il est de bon ton d'apprendre à chanter et à jouer d'un instrument de musique, et où la musique se démocratise tandis que la pratique musicale se privatise, l'introduction du métronome, «dont l'usage est maintenant connu des amateurs $"{ }^{59}$, contribue à alimenter la culture de l'autodidacte. Si aucune allusion au musicien professionnel n'est faite au sein du rapport de l'Académie en faveur du métronome, une attention particulière est, en revanche, accordée à l'amateur : "Un des grands avantages de cette admirable invention est d'inculquer la mesure dans la tête du moins sensible des écoliers élèves»» ${ }^{60}$. Nous saisissons mieux ici pourquoi les académiciens se réclament de la philanthropie pour favoriser la mise sur le marché d'instruments inhérents à la pratique musicale ${ }^{61}$. Or, à mesure que l'art musical est cultivé par le plus grand nombre, le clivage se creuse entre une culture musicale savante et populaire, d'un côté, et entre une pratique musicale professionnelle et amateur, de l'autre. D'une part, le goût musical devient un enjeu social en mesure d'alimenter le désir de promotion et de distinction d'un certain groupe d'auditeurs $^{62}$. D'autre part, la société-post-révolutionnaire dispose d'institutions musicales en mesure de former des musiciens professionnels d'autant plus soucieux de se distinguer des amateurs que le marché musical s'élargit ${ }^{63}$ et que le statut social du musicien évolue ${ }^{64}$. À partir de 1830 , la musique de chambre revient entre les mains des "spécialistes" tandis que les amateurs, qui pratiquent la musique sans en faire un métier et «manifestent leur individualité surtout par leur défauts ${ }^{65}$ ", se transforment progressivement en mélomanes se contentant de l'écouter. Ainsi, à mesure que le métronome est mis à la

58. «Lettre d'Alexis de Lvoff à Fétis [53-14], Saint-Pétersbourg, 17 novembre 1853» in François-Joseph Fétis, Correspondances, op. cit., p. 333. [Autogr.. St-, MLS, D. Frylk. Arch., nº 311.]

59. François-Joseph Fétis, La musique..., op. cit., chapitre 6.

60. BnF, Musique, La. 69 (Début) Bob 21341, n. 195.

61. Sur le rapport ambigu entre formation, institutions musicales et philanthropie, $c f$. Remy Campos, Instituer la musique : les premières années du conservatoire de musique de Genève (1835-1859), Genève, Librairie Droz, 2003.

62. Sur la séparation croissante du peuple et du public au cours du XIX ${ }^{\mathrm{e}}$ siècle, voir Sophie-Anne Leterrier, "Musique populaire et musique savante au XIX" siècle. Du "peuple" au "public" ", Revue d'histoire du XIX siècle, $\mathrm{n}^{\circ} 19,1999$.

63. Sur la mutation que subit l'activité de mécénat à partir des années 1770 , période marquée par le foisonnement d'institutions de concerts publics ou semi-publics, par l'élargissement du marché musical ainsi que par la fermeture des orchestres privés traditionnellement grassement entretenus par les aristocrates les plus fortunés, voir David Hennebelle, "Nobles, musique et musiciens à Paris à la fin de l'Ancien Régime : Les transformations d'un patronage séculaire (1760-1780)", Revue de Musicologie, tome $87, \mathrm{n}^{\circ} 2,2001$, p. 395-418.

64. Alfred Einstein, La musique romantique, Paris, Gallimard, 1959.

65. Jean-Noël Fauquet, Les sociétés de musique de chambre à Paris de la restauration à 1870, Paris, Aux amateurs de livres, 1986, p. 20. 
disposition de l'amateur, plusieurs professionnels - Beethoven en tête - ayant jadis fait l'apologie du métronome, commencent à émettre des réserves : «Pas de métronome! Celui qui a un sentiment juste n'en a pas besoin. Quant à celui qui en est dépourvu, le métronome ne lui sera d'aucune utilité, il fera courir par là tout l'orchestre " ${ }^{66}$. De même, aussitôt après avoir incité les chefs d'orchestre à faire systématiquement usage du métronome, Berlioz les invite plutôt à la modération : «Je ne veux pas dire par là qu'il faille imiter la régularité mathématique du métronome; toute musique exécutée de la sorte serait d'une froideur glaciale, [d'une] plate uniformité» ${ }^{67}$. En revendiquant son autonomie vis-à-vis du métronome et en témoignant de son autosuffisance, le musicien professionnel cherche à se distinguer de l'amateur pour qui l'objet technique s'avère être indispensable. L'article suivant évoque avec fierté la façon dont l'orchestre du Théâtre-italien est parvenu à surmonter les obstacles tendus par le rapide finale du Barbier de Séville de Rossini et ce, sans avoir eu recours au métronome. Le défi musical relevé par l'orchestre est ici présenté comme une victoire de l'homme sur la mécanique.

Nos symphonistes ont résolu encore une fois le problème du finale du Barbier de Seville [...] j'entendois dire à mes cotés : On l'a pris trop vite, ils n'arriveront pas à la fin. Croit-on que l'armée que M. Grasset commande [...] ait besoin d'un métronome, vrai joujou de cabinet, pour régler sa marche? Non! C'est le sentiment qui la guide, c'est la conscience musicale, moyen plus sûr que tous les pendules imaginables. Cet enthousiasme [...] n'a pas fait presser le mouvement d'une seconde ${ }^{68}$.

Les deux pratiques musicales, celle de l'amateur et celle du professionnel, se disjoignent et s'affrontent autour de la question du métronome et de son usage. En effet, utiliser le métronome en qualité de musicien professionnel signifie savoir s'en détacher au moment opportun :

Le métronome est un instrument fort utile pour vous donner la moyenne du mouvement et vous empêcher de vous en écarter grossièrement. Mais quand votre sentiment n'est pas d'accord avec lui, n'hésitez pas à le mettre de côté, car votre propre sentiment, bon ou mauvais, vaudra certainement mieux que ses froides indications qui ne sont que des approximations ${ }^{69}$.

Le musicien professionnel va prendre une position plus nette encore, celle du virtuose. À l'instar du métronome, le piano illustre admirablement cette

66. Anton Schindler, Biographie von Ludwig von Beethoven, Münster, 4/1860, Bd. II, p. 249-250.

67. Hector Berlioz, «Le chef d'orchestre : théorie de son art» in Grand Traité d'instrumentation... op.cit., 1855 .

68. Journal des débats politiques et littéraires, 22 novembre 1823.

69. Lettre d'Édouard Colonne à un musicien, 22 juillet 1882, Aix-les-Bains, lettre autographe n. 245 cité dans Malou Haine, Quatre cent lettres de musicien au Musée royal de Montmarie, Bruxelles, Éditions Mardaga, 1995, p. 395. 
dichotomie socio-culturelle, car il devient non seulement l'enjeu du dilettantisme bourgeois qui se matérialise sous forme de «musique d'ameublement ${ }^{70}$ " à travers l'essor des pianos mécaniques mais aussi le tremplin de la virtuosité romantique. Contrairement à l'amateur, le virtuose peut délibérément s'exercer à la perte de soi et des règles à travers des pratiques telles que la sprezzatura ou le rubato qui délivrent la phrase musicale du carcan du tempo métronomique indiqué et participent ainsi d'un mouvement de désapprentissage. La transgression ne représente-t-elle donc pas pour le virtuose un moyen de se démarquer de l'amateur? La technique entendue comme critère esthétique, ne demande qu'à être dépassée au profit de l'expression sans laquelle la musique se limite à la sphère des arts mécaniques et l'interprète, à un simple artisan. Au contraire, le musicien virtuose "possède des qualités mécaniques qu'il faut vivifier par un sentiment propre $^{71}$ » ce qui implique qu'il est appelé à dépasser le savoir faire et la technique pour produire du "beau» : «il ne faudrait pas avoir pour le métronome une obéissance aveugle; on risquerait fort de jouer comme une mécanique. Cet instrument donne de bonnes indications, et voilà tout; encore faut-il les soumettre au contrôle du sentiment musical, si on en est doué» ${ }^{72}$. En réalité, la virtuosité romantique est tributaire de la nouvelle technique tout en prétendant être son principal adversaire. La nouvelle condition sociale du musicien professionnel de plus en plus livré à lui-même et soumis aux lois du marché nourrit le mythe de l'artiste libre autant qu'il le déconstruit en faisant de la virtuosité romantique un pur produit de l'ère industrielle au même titre que la mécanique. C'est ce que l'écrivain et compositeur Heine dénonce en 1843 :

Cette rage universelle de tapoter sur le piano, et surtout les tournées triomphales des virtuoses $[\ldots]$ marquent spécialement la victoire des arts mécaniques sur l'esprit. La perfection technique, la précision d'un automate, l'identification du musicien avec le bois tendu des cordes, la transformation de l'homme en instrument sonore, voilà ce qui est aujourd'hui prôné et exalté comme le comble de l'art ${ }^{73}$.

70. Manuel Charpy, Le théatre des objets. Espaces privés, culture matérielle et identité sociale. Paris, 18301914. Thèse d'histoire contemporaine, dirigée par Jean-Luc Pinol, Université François-Rabelais de Tours, 2010, Chapitre 7, «Miniaturiser, copier et reproduire : les espaces domestiques et les récits des arts industriels".

71. Journal des débats politiques et littéraires, 22 novembre 1823.

72. Louis-Casimir Colomb, op. cit., p. 53.

73. «Lettre adressée à Berlioz en 1843 " in Heinrich Heine, Lutèce : Lettres sur la vie politique, artistique et sociale de la France, LIV, Genève, Slatkine, 1979, p. 305. 


\section{Conclusion}

Dans son histoire sociale et économique du piano ${ }^{74}$, Cyril Ehrlich se livre à la réflexion suivante : les compositeurs ont-ils un impact sur l'évolution de la facture instrumentale? Est-ce, au contraire, la nouvelle technique qui détermine l'évolution du style compositionnel? Peut-on par ailleurs diviser les compositeurs en deux catégories : ceux qui se contentent de s'adapter à la nouvelle technique et les grands maitres qui en contrôlent et en commandent l'évolution? Nous répondrons que derrière chaque évolution technique relative à la pratique musicale se trouve un grand compositeur en mesure d'exercer tour à tour son autorité sur le mécanicien ou le luthier, sur l'interprète, sur l'amateur, sur ses pairs ainsi que sur l'auditeur. L'action menée par les intermédiaires s'avère cruciale et confirme l'idée selon laquelle «Il n’y a jamais de musique, il n'y a que des montreurs de musique " 75 . Lully fut donc au chronomètre de Loulié ce que Beethoven est au métronome de Maëlzel et ce que Berlioz sera au saxophone de Sax ${ }^{76}$. Or, si le pouvoir «exécutif» du grand compositeur est exacerbé par la double illusion d'objectivité et d'universalité de l'interprétation musicale que lui confere la mécanique, de son côté, l'interprète décline peu à peu le rôle d'exécutant qui lui est concédé au profit d'une affirmation totale de soi, d'où l'émergence d'un nouvel acteur au sein du champ musical : le compositeur-virtuose romantique.

\section{Aurélie Barbuscia est doctorante en histoire contemporaine à l'Institut universitaire européen de Florence.}

\footnotetext{
74. Cyril Ehrlich, The piano..., op. cit., p. 22.

75. Antoine Hénnion, La passion musicale, Paris, Métailié, 1993, p. 296.

76. Le compositeur Berlioz offre d'emblée un soutien inconditionnel au facteur d'instruments et donne une visibilité incomparable au saxophone breveté à Paris en 1846. Dans ses articles comme dans son Traité d'instrumentation, Berlioz ne manque pas de louer les vertus des instruments perfectionnés ou inventés par A. Sax, voir Hector Berlioz, "article du 13 mars 1842» et "notice du 12 juin 1842, Le Journal des Débats» in Critique musicale (1842-1844), édition critique préparée et annotée par Anne Bongrain et Marie-Hélène Coudroy-Saghaï, Paris, Buchet-Chastel, vol. 5, 2004, p. 61 et p. 136-139. Grand traité d'instrumentation et d'orchestration modernes, Cassel, Bärenreiter, 2003.
} 